\title{
APPROACH OF THE INTRACOCHLEAR ACOUSTIC REFLEX IN THE GUINEA PIG
}

\author{
F. DEVRIERE, A. DANCER and P. VASSOUT \\ Département Physiologie, Institut Franco-Allemand de Recherches de Saint-Louis, 5 rue de l'Industrie, \\ BP. 34, F-68301 Saint-Louis cedex, France
}

\begin{abstract}
Groups of anaesthetized guinea pigs were exposed to the same number of impulses $(8 \mathrm{kHz}$ tone-bursts, $10 \mathrm{~ms}$ duration) presented either $17 / \mathrm{s}$ during $1 \mathrm{~min}$ or $1 / \mathrm{s}$ during $17 \mathrm{~min}$. The level of the impulses ranged from 120 to $136 \mathrm{~dB}$ SPL. For a given level both exposure conditions contained the same acoustic energy. The threshold shifts were measured by electrocochleography (from 4 to $20 \mathrm{kHz}$ ) $20 \mathrm{~min}$ after the end of the exposure. Some groups of animals were curarized and some others had the tendons of the middle ear muscles cut in order to eliminate a possible effect of the acoustic reflex of the middle ear. The threshold shifts induced by the $1 \mathrm{~min}$ exposures (17 tone-bursts/s) were always significantly lower than those induced by the 17 min exposures ( 1 tone-burst/s). These results indicate that it exists an "intracochlear acoustic reflex". This reflex could be controlled by the efferent innervation of the cochlea. It is also possible that the intracochlear acoustic pressure acts directly on the outer hair cells and induces their contraction and, by this way, a protection of the cochlear structures. More detailed work is necessary to determine the corrective factors to apply to the Damage Risk Criteria for impulse noises concerning this specific problem.
\end{abstract}

\section{Introduction}

Eight years ago we studied in the guinea pig the threshold shifts (TS) induced by a given number of noise bursts, as a function of their repetition rate (RR) [1]. Following Ward [2], we observed that the maximum TS occurred for a RR of $1 / \mathrm{s}$. The same experiment performed on anesthetized and curarized animals showed no change and indicated that this effect was not due to the acoustic reflex of the middle ear. At this time we proposed that "there might be... protective mechanisms in the cochlea to minimize the mechanical effect of stimulation on the organ of Corti...". Now we will present more detailed results relative to these phenomena.

\section{Methods}

We applied tone bursts of $8 \mathrm{kHz}(10 \mathrm{~ms}$ duration) in closed circuit to the left ear of pigmented guinea pigs anesthetized with Ketamine. The tone-bursts were presented either with a RR of $1 / \mathrm{s}$ during 17 min or with a RR of 17/s during $1 \mathrm{~min}$ (this represents isoenergetic exposures of 10 seconds in total effective duration). The duration of the tone-bursts $(10 \mathrm{~ms})$ was choosen to be shorter that the latency of the efferent fiber response as recorded by Liberman [3], Robertson and Gummer [4]. The RR of 1/s is supposed to give time to the cochlea to return to its original condition before the occurence of the following tone-burst. By this way and as a first approximation tone-bursts presented at a RR of $1 / \mathrm{s}$ can be considered as isolated stimuli applied on a cochlea in which no significant efferent-induced phenomenon exists. On the opposite, a RR of 17/s could correspond to a condition in which the acoustic stimuli are applied on a cochlea in which the protective mechanisms are fully efficient.

Amplitude of the TS are measured ipsilaterally by electrocochleography from 4 to $20 \mathrm{kHz}$ (with an electrode on the round window) twenty minutes after the end of the exposure. In order to insure that the middle ear acoustic reflex did not interfer with our results, we realized the same experiments (1) on anaesthetized and curarized animals, (2) on animals of which the tendons of the middle ear muscles had been cut. 


\section{Results}

On the fig. 1 we can compare the mean TSs induced by the exposure to tone-bursts presented at different stimulation levels ( 120 to $136 \mathrm{~dB}$ SPL), either with a RR of $1 / \mathrm{s}$ during $17 \mathrm{~min}$ (left graph) or with a RR of 17/s during $1 \mathrm{~min}$ (right graph).

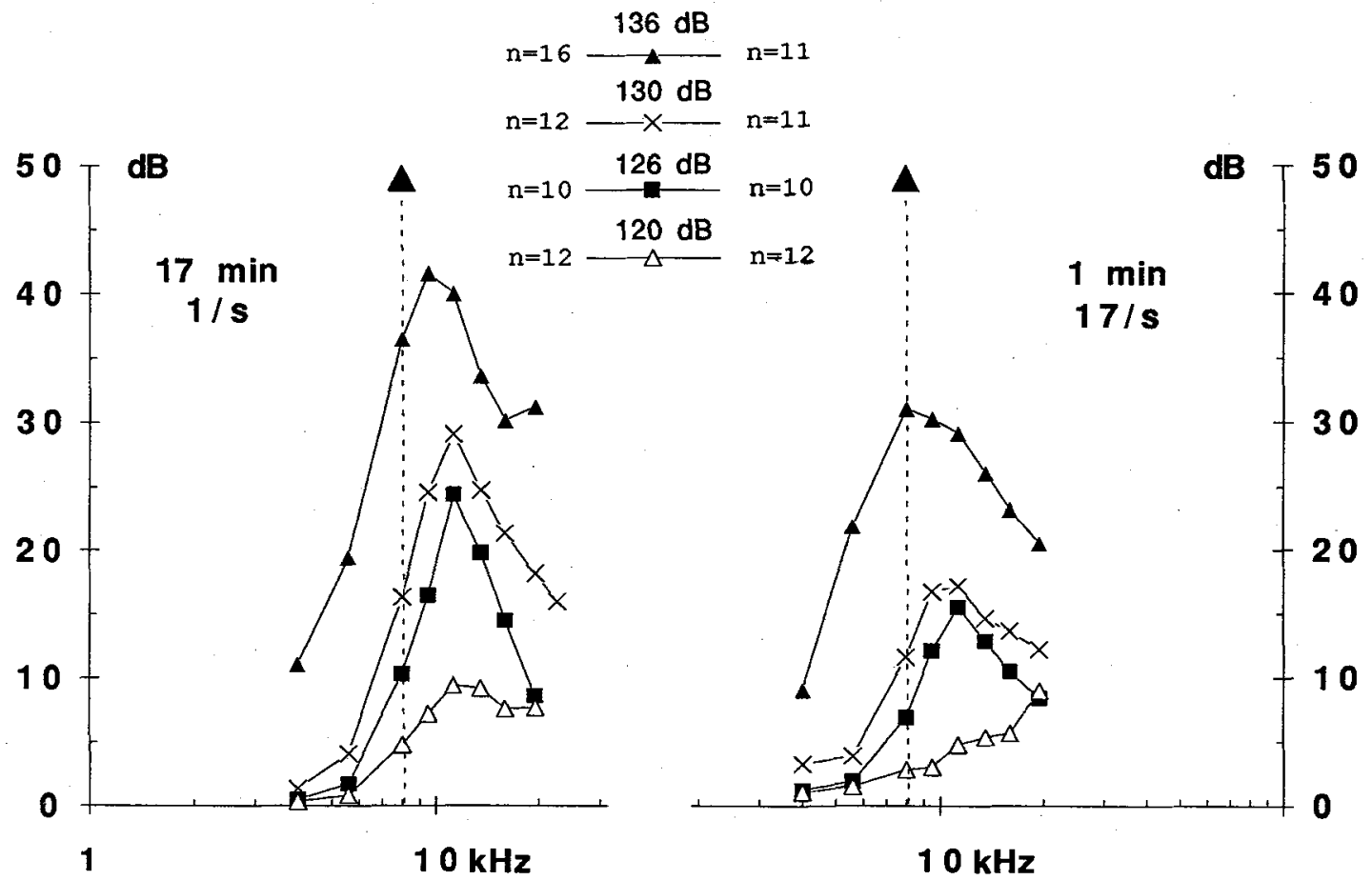

Figure 1. Mean TSs (in $\mathrm{dB}$ ) induced by the exposure to the $8 \mathrm{kHz}$ tone-bursts as a function of the audiometric frequency (in $\mathrm{kHz}$ ) and of the stimulation level (dB SPL). Left Graph: RR 1/s, exposure duration $17 \mathrm{~min}$; Right Graph: RR 17/s, exposure duration $1 \mathrm{~min}$.

(" $n$ " indicates the number of animals in each group)

For each stimulation level, the TSs are smaller (by about $10 \mathrm{~dB}$ ) when the RR is $17 / \mathrm{s}$ : the ear seems to be less susceptible when the RR is high. The difference in susceptibility corresponds at most to a difference of $6 \mathrm{~dB}$ in the stimulation level. Given the frequency of the tone-bursts $(8 \mathrm{kHz})$, and the fact that the guinea pigs were deeply anaesthetized, the middle ear acoustic reflex shouldn't play any role (especially in this species [5]). Nevertheless, we tried to settle this point in two different ways (i) by injecting curare on anaesthetized animals exposed at a RR of $17 / \mathrm{s}$, (ii) by cutting the tendons of the middle ear muscles just before the exposure to tone-bursts of $136 \mathrm{~dB}$ SPL at RRs of $1 / \mathrm{s}$ and 17/s.

(i) In the first experiment, no significant modification of the responses could be observed on the curarized animals.

(ii) In the second experiment (fig. 2) we observed no significant modification of the TSs for a RR of 17/s, but a significant increase of the TSs for a RR of $1 / \mathrm{s}$. If the middle ear muscles were responsible for the differences in susceptibility which we observed on fig. 1, cutting them should modify the TSs much more when the tone-bursts are presented with a RR of $17 / \mathrm{s}$ than when they are presented with a RR of $1 / \mathrm{s}$. The tonicity of the middle ear muscles and its effects on the mechanics of the middle ear (even when the muscles are not activated) could explain these results.

From these two experiments we can conclude that the larger susceptibility of the ear when exposed to tonebursts presented with a RR of $1 / \mathrm{s}$ is not due to the activation of the middle ear mucles.

group and we can exclude an influence of the middle ear muscles. 


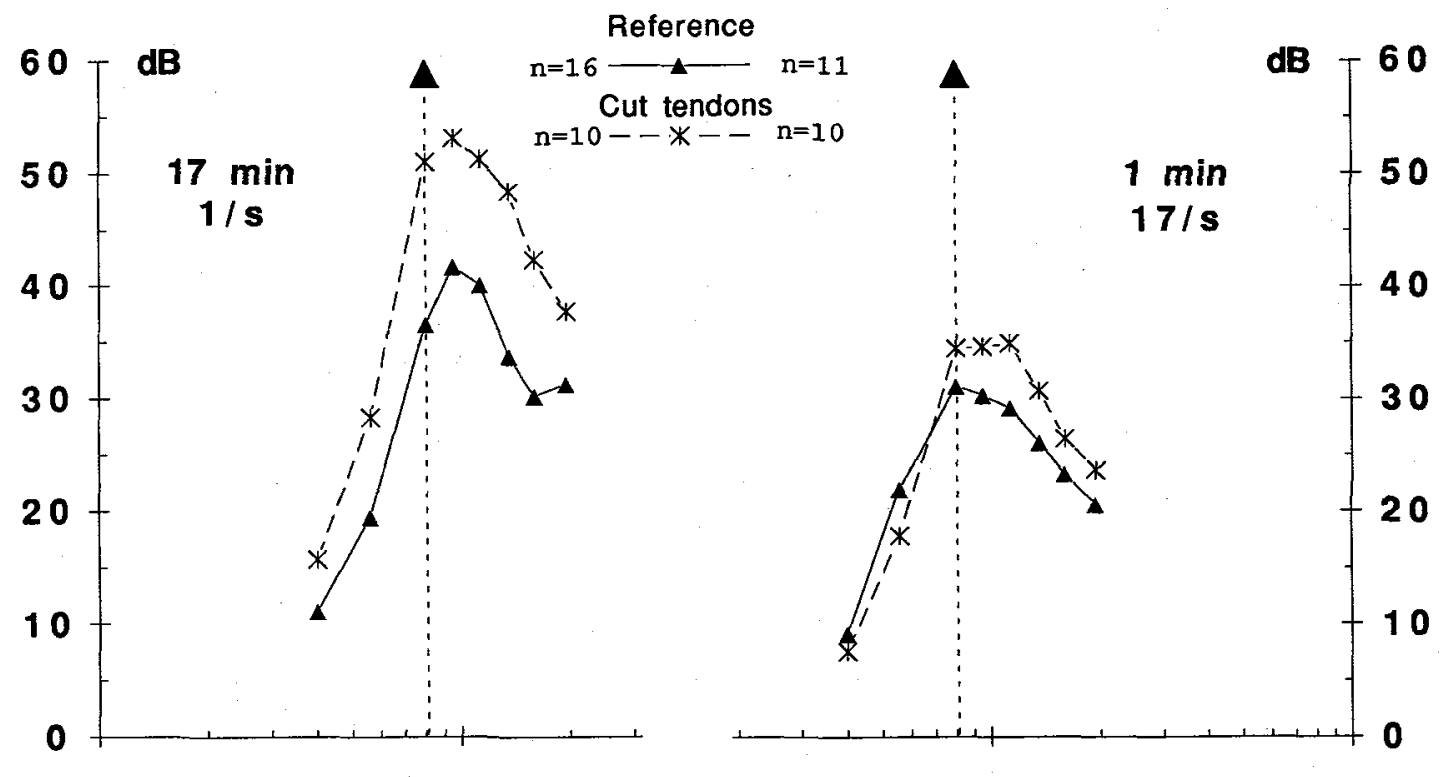

Figure 2. Mean TSs (in $\mathrm{dB}$ ) induced by the exposure to the $8 \mathrm{kHz}$ tone-bursts at $136 \mathrm{~dB}$ SPL in normal animals (reference) and in animals of which the middle ear muscles had been cut (cut tendons). Left Graph: RR 1/s, 17 min; Right Graph: RR 17/s, 1 min. (" $n$ " indicates the number of animals in each group)

In order to investigate a possible protective effect of the median efferent system at the cochlear level, we used two different drugs: scopolamine and strychnine which are known to interfer with the normal behaviour of the efferent system. These drugs were injected to anaesthetized animals 15 to 20 minutes before exposure. On the animals exposed to the tone-bursts at a RR of $17 / \mathrm{s}$ we observed an increase of the TSs. However, the TSs were always smaller than those measured on the animals exposed at a RR of $1 / \mathrm{s}[6]$. One can speculate whether the drugs used in this experiment and the dose we gave to the animals are well adapted to inhibit all the connexions of the efferent system?

The protective effect of the efferent system against high level acoustic stimuli is a very controversial question. According to Liberman [7], in the cat the efferent system does not play any role in protecting the ear from acoustic injury, whereas Rajan [8], in the guinea pig, fosters the opposite statement.

It is also possible that the outer hair cells are directly and mechanically excited by the acoustic stimulus, especially at such high intensities, and are able to respond by contracting themselves and limiting the mechanical input to the Corti's organ (without the action of the efferent bundle) [9],[10].

On the other hand it is interesting to note that the TSs induced by the exposure to tone-bursts do not exhibit the same characteristics as those induced by the exposure to a continuous pure tone of the same frequency $(8 \mathrm{kHz})$. When guinea pigs are exposed to a $8 \mathrm{kHz}$ pure tone during $20 \mathrm{~min}$, the maximum TS is located at a higher frequency (at moderate levels one can observe the well-known phenomenon of the half-octave shift), and the size of the shift increases with the stimulation level and the amount of the TS [11]. In the experiments reported above (tone-bursts exposures, see fig. 1) the size of the shift has a tendency to decrease with the stimulation level and the amount of TS. On the figure 3 we have plotted the value of the ratio between the frequency of the maximum TS and the stimulus frequency, as a function (i) of the stimulus level, (ii) of the amplitude of the maximum TS, both for continuous tone exposures (8 $\mathrm{kHz}, 20 \mathrm{~min}, 85$ to $123 \mathrm{~dB} \mathrm{SPL})$ and for tone-bursts exposures $(8 \mathrm{kHz}, 10 \mathrm{~ms}, 1 / \mathrm{s}$ for 17 . min and 17/s for $1 \mathrm{~min}$ ). The differences are striking and could indiquate that the fatigue and/or the injury mechanisms are not the same whether the ear is exposed to continuous or to impulse noises. The pattern of the TSs recorded $20 \mathrm{~min}$ after the end of the exposure to the tone-bursts looks like this one of the permanent threshold shifts (PTS) measured some days after the exposure to pure tones (at the end of the recovery). 


\section{Fmax/Fstim}
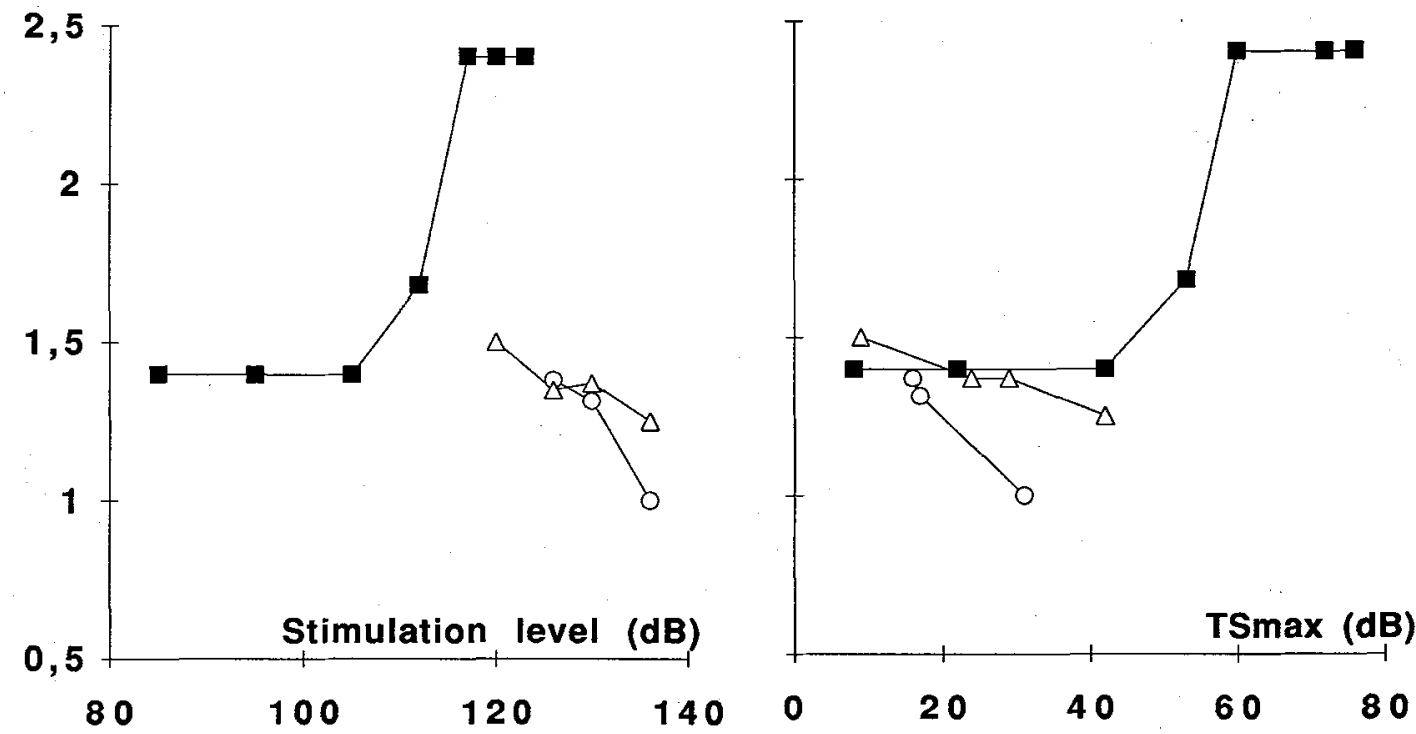

Figure 3. Ratio between the frequency of the maximum TS and the stimulus frequency (Fmax/Fstim) as a function (i) of the stimulus level (left graph), (ii) of the amplitude of the maximum TS (right graph), for continuous tone exposures ( $8 \mathrm{kHz}$ for $20 \mathrm{~min}: \longrightarrow \longrightarrow$ ) and for tone-bursts exposures $(8 \mathrm{kHz}, 10 \mathrm{~ms}, 1 / \mathrm{s}$ for $17 \mathrm{~min}: \triangle-\triangle \quad$ and $17 / \mathrm{s}$ for $1 \mathrm{~min}:-0-$ )

\section{Conclusion}

The results indicate that protective mechanism(s) against acoustic injury exist(s) in the cochlea. This mechanism could be mediated by the efferent system. It could also be due to a direct acoustical/mechanical excitation of the outer hair cells. Following an acoustic stimulation the outer hair cells would contract themselves and reduce their dynamical range as well as their susceptibility to the acoustic stimulus by about $6 \mathrm{~dB}$.

This phenomenon should be taken into account by the Damage Risk Criteria for impulse noises and more detailed work is necessary to determine the corrective factors to apply to these criteria concerning this specific problem.

\section{References}

[1] Buck, K., Dancer, A. and Franke, R., J. Acoust. Soc. Am., 76, (1984), 1090-1097.

[2] Ward, W.D., J. Acoust. Soc. Am., 34, (1962), 1230-1232.

[3] Liberman, Ch., J. of Neurophysiology, 60, (1988), 1779-1798.

[4] Robertson, D. and Gummer M., Hearing Res., 20, (1985), 63-77.

[5] Avan, P., Hearing Res., (1992), to be published.

[6] Vassout, P., Dancer, A. and Rebillard, G., 13th Midwinter Meeting of the A.R.O., St. Petersburgh, (1990), 324.

[7] Liberman, Ch., Noise-Induced Hearing Loss, eds., A. Dancer et al., Mosby Year Book, (1991), 423-428.

[8] Rajan, R., Noise-Induced Hearing Loss, eds., A. Dancer et al., Mosby Year Book, (1991), 429-444.

[9] Brundin, L., Flock, A. and Canlon, B.Nature, (1989), 814.

[10] Ding, J.P., Salvi, R.J. and Sachs, F., 13th Midwinter Meeting of the A.R.O., St Petersburgh, (1990), 226.

[11] Décory, L., Dancer, A. and Aran, J.M., Noise-Induced Hearing Loss, eds., A. Dancer et al., Mosby Year Book, (1991), 73-88. 